\title{
Automatic Feature Extraction of Pose-measuring System Based on Geometric Invariants
}

\author{
Yan Lin ${ }^{1,2}$ Bin Kong ${ }^{2}$ Fei Zheng ${ }^{2}$ \\ ${ }^{1}$ Center for Biomimetic Sensing and Control Research, Institute of Intelligent Machines, Chinese Academy of \\ Sciences, Hefei 230034, P.R. China \\ ${ }^{2}$ Department of Automation, University of Science and Technology of China, Hefei 230027, P.R. China
}

\begin{abstract}
This paper proposes a method for extracting features of target object automatically in a pose-measuring system. A tag stuck to the target object is designed to make the system more robust and precise. First, detect all the corners of an image. Then, geometric invariants, which are formed by several invariants of four collinear points, are used to distinguished corners of the tag from all the corners. To alleviate the computational complexity, polar coordinate system is introduced for searching collinear points. Experimental results show that this method can extract features of the target automatically, rapidly and exactly under different viewpoints.
\end{abstract}

Keywords: Geometric invariant, Perspective invariant, Feature extraction, Pose-measuring system

\section{Introduction}

With the development of technology of robot, it becomes important to obtain the relationship between two objects rapidly and exactly, so that the objects can correspond with each other. Take robotic assembly in the industry for example, relationship of position and pose between the robot and the component to be assembled is needed to control the robot grasping the component. Computer vision is used commonly in pose-measuring system. Generally, one or two cameras are placed on one object (observer), which acquire images of the other object(target object). By searching out the fixed features of the target object in 2-D images, and then calculate the 3-D coordinate of these features in the observed coordinate system. Finally, the pose of target object relative to the observed coordinate system can be obtained [1]-[2].

In order to construct a stable and precise pose-measuring system, feature extraction of the target object is a key step [3]. In some situation, inconspicuous features of target object will bring difficulty to the pose estimation. Usually, a man-made tag is provided to the target, so that the feature extraction will be easier, and the system will become more robust.
The projective transform, which occurs in the 2-D imaging process, causes unavoidable difficulty, since it produces different images, according to the viewpoint of the camera [4]. However, the geometric invariants are known to provide effective solutions for these problems, since the geometric invariants possess the properties which are invariant to the viewpoints. Thus the geometric invariants received considerable interests in computer vision research recently. Several geometric invariants have been proposed, including algebraic invariants [5] and differential invariants [6]. Among the well-known geometric invariants, the invariants of "four collinear points", "five coplanar points" and "five coplanar lines" are the most easily applicable ones [7]-[8].

In this paper, a chessboard tag is stuck to the target object to make the system more stable and precise. Then the features of the tag served as the features of target object. Corner is selected as features to be detected. Geometric invariants of the tag are composed of the invariants of "four collinear points". By using geometric invariants, corners of the chessboard tag can be located exactly under different viewpoints. When search for collinear points, polar coordinate system is established to alleviate the computational complexity. Finally, real images took by Binocular Pose-measuring System under different viewpoints were tested. The results show the feasibility of the proposed method.

\section{Automatic feature extraction of the image}

\subsection{Selection of the feature}

The efficiency and precision of the measurement system will be influenced by the type of feature. Corners not only reserve important information of image, but also effectively decrease the amount of information. So corner is selected as feature in this method. Corners are widely used for image alignment, $3 \mathrm{D}$ reconstruction, Motion tracking, Object recognition, Robot navigation, and so on.

Harris corner detector [9] is selected to extract features of a whole image. It can extract corners 
exactly due to its invariance to: rotation, scale, illumination variation and image noise [10], so the stability of the system can be guaranteed.

\subsection{Design of the tag}

In actual measuring system, the target object is always provided with a tag in order to find it more easily. Chessboard is a widely used template in camera calibration. Take a small size chessboard as the tag, as shown in Fig.1 (a) . There is a certain distance between the border of the tag and the chessboard. The size of the tag can be adjusted according to request. Due to the man-made tag, the signal-to-noise ratio is enlarged. Thus the influence of the measurement noise is decreased.

Corner is usually defined as the point of maximal planar curvature in the line of the steepest gray-level slope. The corners of the chessboard label is shown by red crosses in Fig.1 (b).
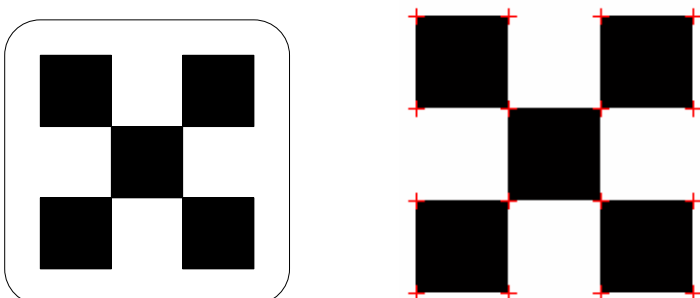

Fig.1: (a) label of chessboard (left) (b) Corners of the chessboard (right).

\section{Detection of the corners of the tag}

After extracting all the corners of the image, information decrease remarkably. Then the corners of the tag can be distinguished based on geometric invariants. When viewpoint varies, the geometric information of chessboard tag is not preserved, such as lengths, angles and parallelism. The imaging process is a perspective projection of points in the world to points in the image plane. So perspective invariants can be used to locate the chessboard tag.

\subsection{Perspective invariants}

Perspective projection is an idealized mathematical model of the behavior of real cameras. As shown in Fig.2, the center of projection is at the origin $O$ of the $3 \mathrm{D}$ reference frame of the space. The image plane $\Pi$ is parallel to the $(\vec{x}, \vec{y})$ plane and displaced a distance $f$ (focal length) along the $\vec{z}$ axis from the origin. The 3D point $P$ projects to the image point $p$. The orthogonal projection of $O$ onto $\Pi$ is the principal point $o$, and the $\vec{Z}$ axis which corresponds to this projection line is the principal axis.

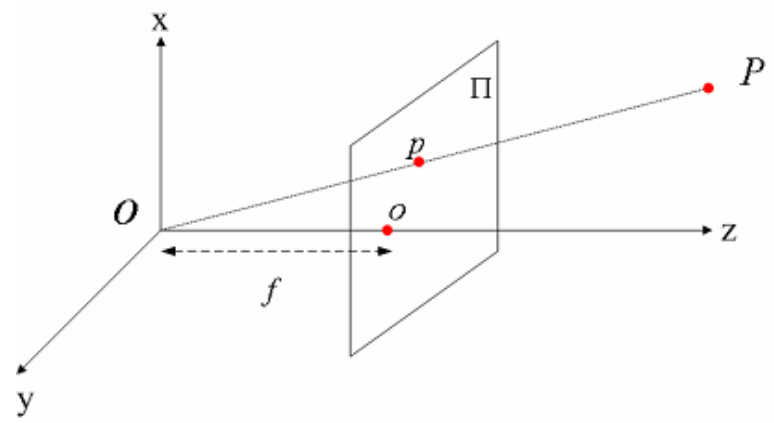

Fig.2: Perspective projection.

Every point in an image represents a possible line of sight of an incoming light ray: any 3D point along the ray projects to the same image point, so only the direction of the ray is relevant, not the distance of the point along it. Suppose there is a point $(x, y)$ in the Euclidean plane, homogeneous coordinates $(X, Y, T)$ is used to represent this same point in the projective plan, then $x=\frac{X}{T}, y=\frac{Y}{T}$.

Suppose there are three collinear points $A\left(X_{1}, Y_{1}, T_{1}\right), B\left(X_{2}, Y_{2}, T_{2}\right), C\left(X_{3}, Y_{3}, T_{3}\right)$ in the projective plan, the determinant of the $3 \times 3$ matrix containing the points is zero:

$$
\left|\begin{array}{l}
X_{1}, Y_{1}, T_{1} \\
X_{2}, Y_{2}, T_{2} \\
X_{3}, Y_{3}, T_{3}
\end{array}\right|=0
$$

Projective geometry preserves neither distances nor ratios of distances. However, the cross ratio, which is a ratio of ratios of distances, is preserved. Given four collinear points $p_{1}, p_{2}, p_{3}$ and $p_{4}$ in the projective plan, denote the Euclidean distance between two points $p_{\mathrm{i}}\left(X_{i}, Y_{i}, T_{i}\right)$ and $p_{\mathrm{j}}\left(X_{j}, Y_{j}, T_{j}\right)$ as $\Delta_{i j}$. Then, one definition of the cross ratio is the following:

$$
\begin{gathered}
\operatorname{Cr}\left(p_{1}, p_{2} ; p_{3}, p_{4}\right)=\frac{\Delta_{13} \Delta_{24}}{\Delta_{14} \Delta_{23}} \\
\Delta_{i j}=\sqrt{\left(\frac{X_{i}}{T_{i}}-\frac{X_{j}}{T_{j}}\right)^{2}+\left(\frac{Y_{i}}{T_{i}}-\frac{Y_{j}}{T_{j}}\right)^{2}}
\end{gathered}
$$

In other words, select one of the points, say $p_{1}$, to be a reference point. Compute the ratio of distances from that point to two other points, say $p_{3}$ and $p_{4}$. Then compute the ratio of distances from the remaining point, in this case $p_{2}$, to the same two points. The ratio of these ratios is invariant under projective transformations. 


\subsection{Location of the chessboard tag}

As shown in Fig.3, there are 16 corners on the chessboard. The numbers of them are ai $(i=1 \sim 16)$. Ten lines with four collinear points can be formed by these corners, as shown by red lines in Fig.3. By projective transformations, four collinear points in the world are still collinear in the image plane, and cross ratio is invariant with the same order of the points.

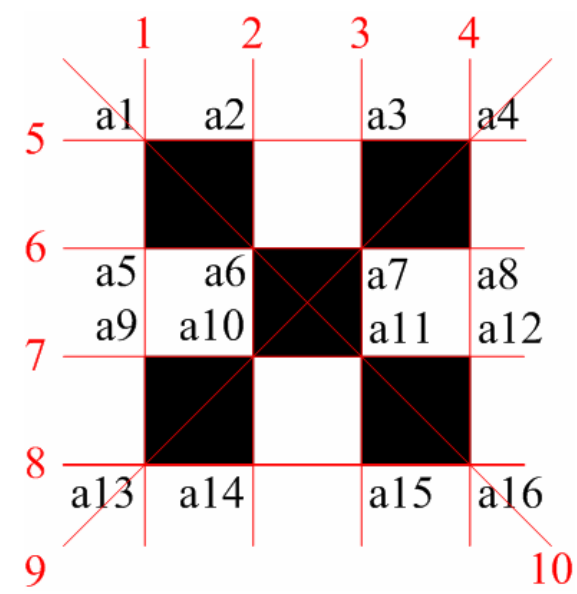

Fig.3: Corners and lines of the chessboard tag.

If these lines, which are formed by four collinear corners, can be detected, corners of the tag can be distinguished. However, as the number of extracted feature increases, the number of candidates grows rapidly, requiring enormous computational loads. To decrease the search space, select the outer corners (corner $a 1, a 4, a 13, a 16$ in Fig. 3 ) as the goal of the search. Then the other corners of the chessboard tag can be found easily. As shown in Fig.3, there are three lines pass through the outer corner, and outer corner lines on one side of the lines. Suppose corner $a i$ is on detecting. If three lines formed by four collinear corners pass through ai can be detected, and cross ratios are right, corner ai maybe outer corner. And the corners on the three line which are farthest from corner ai maybe the other three outer corners.

Searching the collinear corners based on equation (1) requires enormous computational loads. For detect the collinear corners quickly, polar coordinate system with corner on detecting as its pole is established. Its polar axis is parallel to the $\mathrm{x}$-axis of the image. Polar coordinate of a point can be described as $P(r, t) . r$ is the distance between pole and the point. U-axis is radial from the pole to the point. $t$ is the value of the angle from the pole axis to the u-axis, which called polar angle. Suppose there are two points $A\left(r_{1}, t_{1}\right)$ and $B\left(r_{2}, t_{2}\right)$. If $t_{1}=t_{2}$, points $A, B$ and pole are collinear, $A$ and $B$ are on the same side of pole.
As shown in Fig.4 (a); if $\left|t_{1}-t_{2}\right|=\pi$, points $A, B$ and pole are collinear, pole is between points $A$ and $B$. As shown in Fig.4 (b).

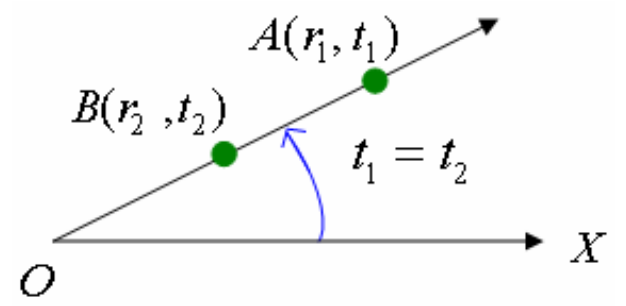

(a) points $A, B$ on the same side of pole

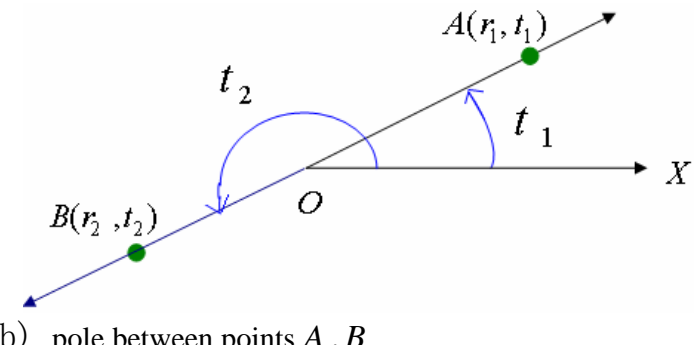

(b) pole between points $A, B$

Fig.4: collinear points in Polar coordinate system.

Practical lens systems of camera are nonlinear and can easily introduce significant distortions of the 2-D image plane. So, collinear points would not be collinear strictly after perspective transformation. Suppose that, the range of $t$ is $0 \leq t<2 \pi$. When $t_{1}$ and $t_{2}$ are equal approximately, points $A, B$ and pole can be judged as collinear, and $A, B$ are on the same size of pole. The definition of collinear is shown by equation (4). threshold 1 is a small value, for example 0.05 radian. It should be considered that, if the u-axes of points $A$ and $B$ are near to pole axis, difference between $t_{1}$ and $t_{2}$ maybe large. For example, points $A\left(r_{1}, 0.02\right)$ and $B\left(r_{2}, 6.26\right)$. In this situation, $t_{1}$ and $t_{2}$ satisfy equation || $0.02-6.26|-2 \pi|<$ threshold 1 .

$$
\begin{aligned}
& \left|t_{1}-t_{2}\right|<\text { threshold1 or } \\
& || t_{1}-t_{2}|-2 \pi|<\text { threshold1 }
\end{aligned}
$$

When calculate the cross ratio of four collinear points, order of the four points should be decided. Suppose the corner on detecting is corner $p_{1}$, corners which are collinear with $p_{1}$ can be found according to equation (4). Calculate the distance between these corners and $p_{1}$, three corners nearest to $p_{1}$ are numbered $p_{2}, p_{3}, p_{4} . p_{2}$ is nearest from $p_{1}$, while $p_{3}$ is the farthest. If $p_{1}$ is outer corner of chessboard, according to equation (3), cross ratio of corners

$$
p_{1}, p_{2}, p_{3}, p_{4}
$$


is $\operatorname{Cr}\left(p_{1}, p_{2} ; p_{3}, p_{4}\right)=\frac{\Delta_{13} \Delta_{24}}{\Delta_{14} \Delta_{23}}=4 / 3$. Considering the distortions, cross ratio satisfies $\quad\left|C r\left(p_{1}, p_{2} ; p_{3}, p_{4}\right)-4 / 3\right|<$ threshold 2 threshold 2 is a small value, for example 0.1 .

\section{Realization of the algorithm}

According to the analysis above, the whole method for processing an image is as follow:

(1).Detect all the corners of an image using Harris corner detector. Record the coordinate of corners in corners[count], count is the total number of the corners.

(2). Detect corners in corners one by one to judge if it is outer corner of chessboard. Suppose the corner on detecting is corners[i] :

(a) Establish a polar coordinate with corners[i] as its pole. Calculate the value of polar angle of other corners. Then record these values and corresponding number of the corners in angle. To alleviate the computational loads, only calculate the corners in a certain area around the pole. The area should be large enough to cover the chessboard.

(b) Sorts the elements of angle in non-decreasing order according to the value of polar angle. Detect the array angle. Search if there are three groups of values which are equal to each other approximately (defined by equation (4)). If not, go to (3). If so, search out three corners nearest to corners $[i]$ in each group, and record the number of corners farthest from corners[i] in variables $a, b, c$. Then calculate the cross ratio of the four collinear corners according as the method presented in section 3.2. If cross ratios are invariable, corners[i] maybe outer corner of chessboard, go to (c).

(c) Detect respectively if corners $a, b, c$ are outer corners. The method is described in (2)-(a) and (2)-(b). If so, four outer corners are found, go to (4). If not, go to (3).

(3). If $i<$ count,$i++$, go to (2);

If $i=$ count , outer corners are not found. End the algorithm.

(4). Locate other corners of the chessboard by four outer corners: Calculate the lines decided by two outer corners. There are six such lines. On each line, there will be two corners between outer corners. Then, the other 12 corners of chessboard can be located.

\section{Experiment and conclusion}

Several pairs of images took by Binocular Pose-measuring System under different viewpoints were tested for validating the feasibility of this method. Several results of detection are shown in Fig.5. Left images are taken by left camera, and right images are taken by right camera. The size of image is $1248 * 1024$. The total number of the images in Fig.5 is about 100, and the algorithm cost less than 300 millisecond. The algorithm is implemented using Visual $\mathrm{C}++6.0$ and OpenCV. The condition of hardware is: CPU, Pentium4 1.8G; Memory, 256M.

In these images, corners of a whole image are shown by the cross. Outer corners of chessboard tag are shown by green cross. Other corners of chessboard are shown by red cross. The coordinates of outer corners of chessboard, total number of corners of images and the time of processing an image are listed in Table1. The origin of image coordinate system is at top left corner of the image. $\mathrm{X}$ represent column-coordinate while $\mathrm{Y}$ represent row-coordinate.

In this paper, a method for automatic feature extraction of Pose-measuring System based on Geometric Invariants was presented. A chessboard tag stuck to the target object improved the precision and robustness of the system. The features of the tag substituted for features of the target object. Geometric invariants were used to distinguish the corners of chessboard from all the corners of an image. The basic invariant of four collinear points enables exact location of chessboard under different viewpoint. To alleviate the computational complexity, polar coordinate system was employed to search collinear points. This method can detect the corners of target object automatically, rapidly and exactly. This method also meets the request of real-time computation.

\begin{tabular}{|c|c|}
\hline & coordinates of outer corners $(\mathrm{X}, \mathrm{Y})$ \\
\hline Fig.5(a1) & $(414,228),(470,95),(538,275),(595,149)$ \\
\hline Fig.5(b1) & $(850,197),(1024,116),(917,63),(957,244)$ \\
\hline Fig.5(a2) & $(319,90),(356,277),(286,181),(395,191)$ \\
\hline Fig.5(b2) & $(764,247),(746,55),(832,159),(684,149)$ \\
\hline Fig.5(a3) & $(334,524),(177,399),(217,523),(298,396)$ \\
\hline Fig.5(b3) & $(773,506),(640,507),(617,382),(755,378)$ \\
\hline
\end{tabular}

Table 1: coordinates of outer corners of chessboard. 


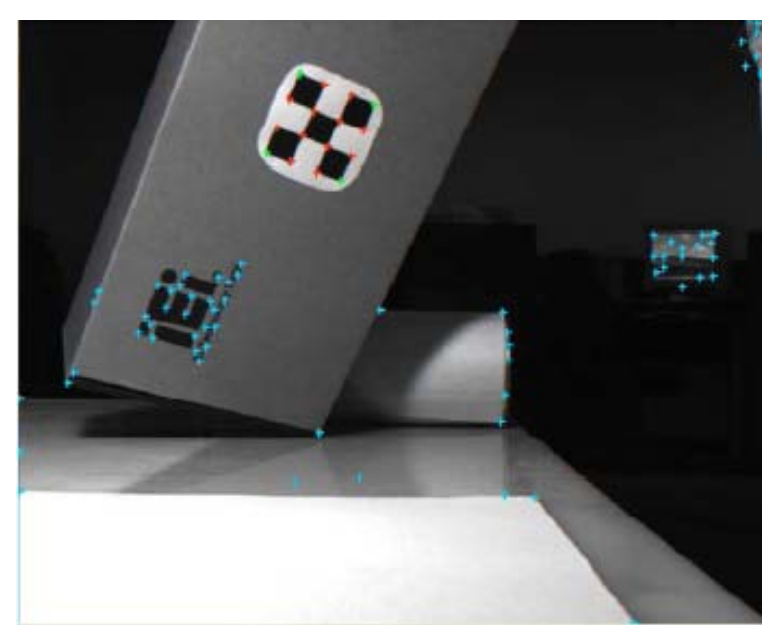

(a1)

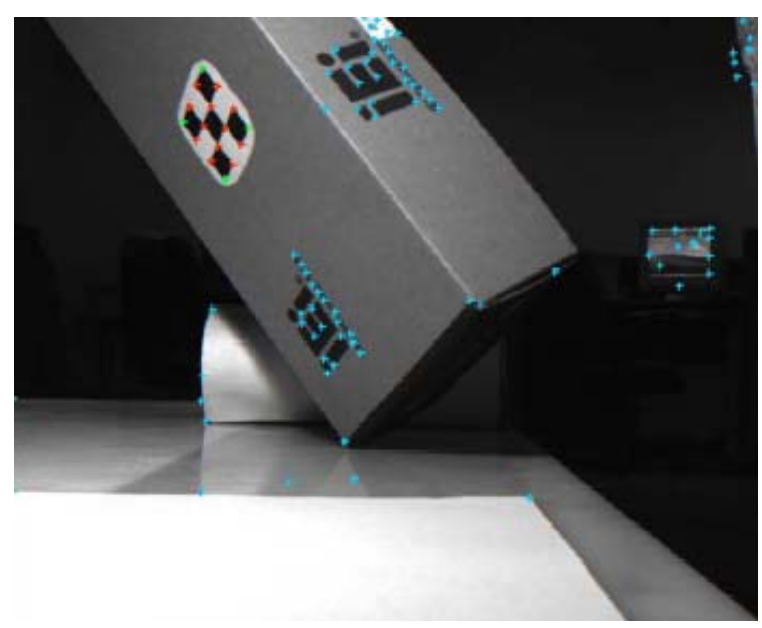

(a2)

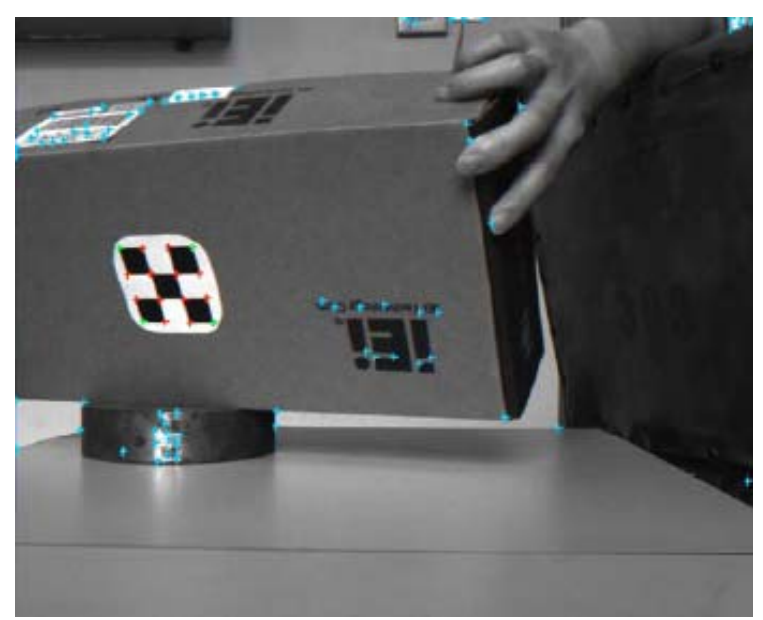

(a3)

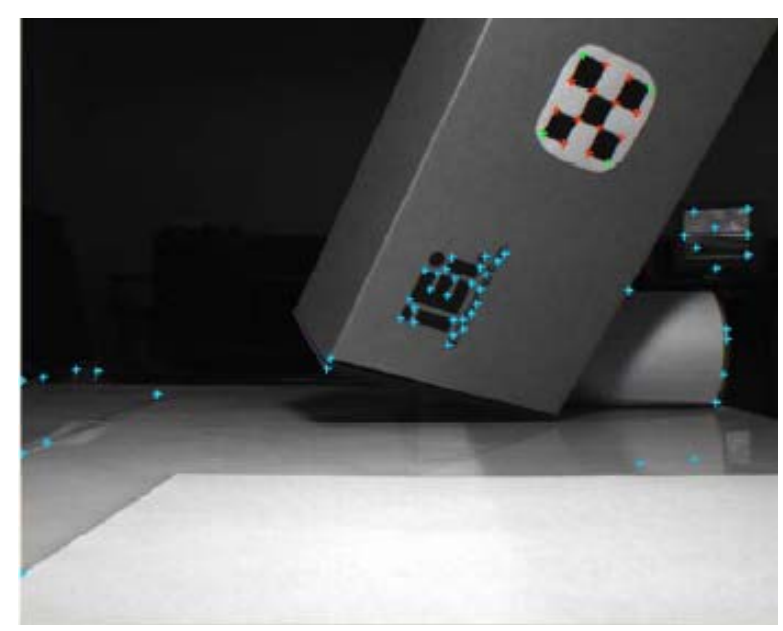

(b1)

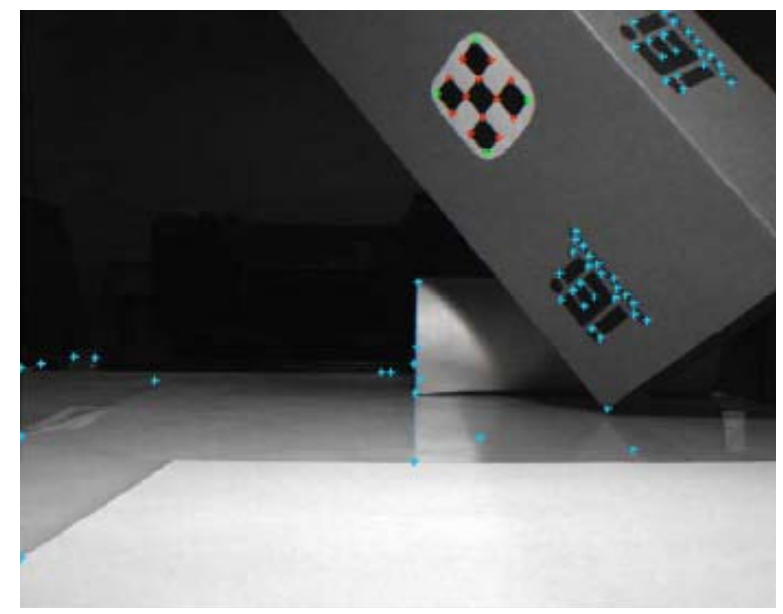

(b2)

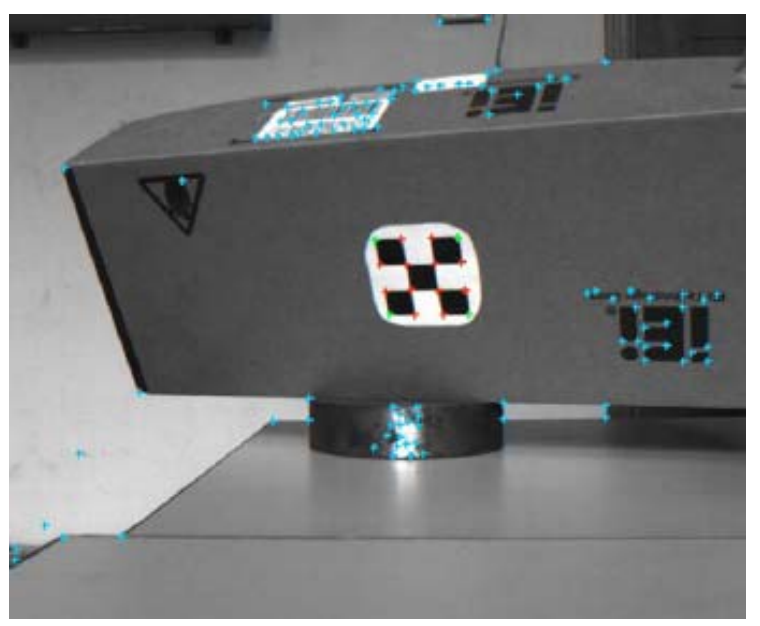

(b3)

Fig.5: results of detection of images under different viewpoints 


\section{Acknowledgement}

This work is supported by National Natural Science Foundation of China (No.60572053), NSF of Anhui province (No.050420307).

\section{References}

[1] G.D. van Albada, J.M. Lagerberg, A.Visser and L.O.Hertzberger. A low-cost pose-measuring system for robot calibration. Robotics and Autonomous Systems, 15: 207-227, 1995.

[2] Y. M. Hao, F. Zhu, J. J. OU. 3D Visual Methods for Object Pose Measurement. Journal of Image and Graphics, Vol. 7(A), No.12 Dec.2002.

[3] J. Usabiaga, A. Erol, G. Bebis and R. Boyle, et.al., Global Hand Pose Estimation by Multiple Camera Ellipse Tracking. 2nd International Symposium on Visual Computing (ISVC06), Lake Tahoe, November 6-8, 2006.

[4] J. L. Mundy, A. Zisserman, Geometric Invariance in Computer Vision, MIT Press, Cambridge, 1992.

[5] S. Carlsson, Projectively invariant decomposition and recognition of planar shapes, Internat. J. Comput Vision, 17(2): 193-209, 1996.

[6] I. Weiss, Geometric invariants and object recognition, Int.J. Comput Vision 10(3): 207-231, 1993.

[7] TomaHs Suk, Jan Flusser. Point-based projective invariants. Pattern Recognition, 33: 251-261, 2000.

[8] Bong Seop Song, Kyoung Mu Lee, Sang Uk Lee. Model-Based Object Recognition Using Geometric Invariants of Points and Lines. Computer Vision and Image Understanding, 84: 361-383, 2001.

[9] C. Harris, M.J. Stephens. A combined corner and edge detector, In Alvey Vision Conference, pp. 147-152, 1988.

[10] C. Schmid, R. Mohr, C. Bauckhage, Evaluation of interest point detectors. International Journal of Computer Vision, 37(2):151-172, 2000. 